To the Editors:

\title{
Outcome of skin sensitivity testing for predicting reactions to rabies equine immunoglobulin
}

Post-exposure prophylaxis effectively prevents rabies after rabid animal bites. Rabies immunoglobulin (RIG) is essential immediately after all category III major bites as vaccination alone takes time to produce an effective antibody response. Of the two RIGs available in Sri Lanka, human RIG is prohibitively expensive but safer. Equine RIG is less expensive but carries a risk of causing allergy and anaphylaxis. Hence a skin sensitivity test (SST) is advised before the administration of equine RIG. It is done by intradermal administration of $0.1 \mathrm{ml}$ of equine RIG in a 1 in 10 dilution with isotonic saline, which would raise a bleb of 3-6mm in diameter. A control test is done on the opposite forearm with $0.1 \mathrm{ml}$ of saline only. Any wheal or flare with induration greater than $10 \mathrm{~mm}$ on the test side is considered a true positive. If both sides give a positive reaction, it is considered as a false positive. If the SST is a true positive, equine RIG is withheld and the patient is prescribed human RIG or the 8-site intradermal regimen. The rate of positive SST has not yet been recorded in Sri Lanka for equine RIG and the aim of this study is to find it.

We collected data from three registries maintained in the outpatient department and preliminary care unit of the Teaching Hospital, Peradeniya. All patients who were given rabies post-exposure prophylaxis from 28 August to 6 November 2006 were included.

Age, gender, outcome of SST (either positive or negative), and manufacturing details of equine RIG were recorded. Data were analysed using the statistical software package SPSS version 10 .

A total of 938 patients had received post-exposure therapy during the study period. Of them $530(56.5 \%)$ had qualified for RIG and had been subjected to SST (SST group) comprising 290 males and 240 females with the median age 19 years (range 1-84) and 25 years (range 1 80 ) respectively. Male to female ratio was 1:0.83. There were $353(66 \%)$ adults and $177(33 \%)$ children below 12 years. A total of $141(26.6 \%)$ were positive for SST comprising $33(18.6 \%)$ children and $108(30.6 \%)$ adults, and none of them had been given equine RIG. The SST negative number was 389 (74.4\%) and all had received equine RIG. Of them, one had developed a mild allergic reaction to RIG with a negative predictive value of $99.8 \%$.
During the study period 537 and 40 vials of equine RIG manufactured respectively by Bharat Serums and Vaccine Limited of India and Changchun Institute of Biological Products of China were used. The average cost of an equine RIG vial is 457 Rupees, which is about onetenth the cost of a human RIG vial.

We found that about one-fourth of patients had a positive SST, thus predicting the cost involved to import human RIG for them, which is a colossal financial burden to a poor nation. Also we found a high negative predictive value of reactions in the RIG treated SST negative patients. But the calculation of positive predictive value of reaction was not a reality in this series. Concerns have been expressed about the sensitivity of SST and its predictive value of reactions [1,2]. In fact, in Sao Paulo, the practice of SST has been abandoned before giving equine RIG [3] . And some highly purified equine RIG preparations had not caused significant allergic reactions following administration [4,5]. Ideally, rabies post-exposure prophylaxis should be done in dedicated anti-rabies units, by specially trained staff to minimise the number of false positive SST. An answer to the problem may be to develop a purified equine RIG without compromising efficacy.

\section{References}

1. Tantawichien J, Benjavongkulchai M, Wilde H, Jaijaroensup W, Siakasem A, Chareonwal S, Yountong C, Sitprija V. Value of skin testing for predicting reactions to equine rabies immune globulin. Clinical Infectious Disease 1995; 21: 660-2.

2. Wilde H, Chomchey P, Prakongsri S, Punyaratabandhu P Safety of equine rabies immune globulin. Lancet 1987; 2: 1275-80.

3. Cupo P, de Azevedo-Marques MM, Sarti W, Hering SE. Proposal for abolition of the skin sensitivity test before equine rabies immune globulin application. Revista do Instituto de Medicina Tropical de Sao Paulo 2001; 43: 51-3.

4. Suwansrinon K, Jaijareonsup $H$, Wilde $M$, Benjavongkulchai C, Sriaroon Sitprija V. Sex and age related differences in rabies immunoglobulin hypersensitivity. Transactions of the Royal Society of Tropical Medicine and Hygiene 2007; 101: 206-8.

5. Wide $\mathrm{H}$, Chutivongse $\mathrm{S}$. Equine rabies immune globulin: a product with an undeserved poor reputation. American Journal of Tropical Medicine and Hygiene 1990; 42: 175-8.

S A M Kularatne (Professor), M C Gihan (Assistant Lecturer), Department of Medicine, Faculty of Medicine, University of Peradeniya; A M Jameel (Senior Medical officer), General Hospital, Peradeniya, Sri Lanka, O Wimalaratne (Virologist), Medical Research Institute, Colombo.

Correspondence: SAMK, e-mail <samkul@sltnet.lk>. Received 25 April, and revised version accepted 17 August 2007. Conflicts of interest: none declared. 